\section{Hypothesis: social defeat is a risk factor}

\section{for schizophrenia?}

JEAN-PAUL SELTEN and ELIZABETH CANTOR-GRAAE really 'begins'. Interestingly, a study of discordant twins has shown that divergence in school performance precedes the onset of psychosis by an average of 10 years (van Oel et al, 2002). This suggests that certain causal factors may operate long before the emergence of psychotic symptoms and that the time frame of life-event studies is inadequate. The purpose of this paper is to present evidence in support of the hypothesis that a chronic and long-term experience of social defeat may lead to sensitisation of the mesolimbic dopamine system (and/or to increased baseline activity of this system) and thereby increase the risk for schizophrenia. The hypothesis is based on epidemiological findings, studies of dopamine function in humans and animal experiments.

\section{EPIDEMIOLOGICAL FINDINGS}

Some established risk factors for schizophrenia, which are not purely genetic, are the urban environment, migration, low IQ, hearing impairment and the use of illicit drugs.

Krabbendam \& van Os (2005) performed a meta-analysis of studies that examined the rate of schizophrenia in urban as compared to rural areas, and obtained a mean weighted relative risk (RR) of 1.72 (95\% CI 1.53-1.92). The available evidence suggests that causation (urban environment causes psychosis) is more important than selection (high-risk individuals move into urban areas) and that the effect of the environmental factors in the urban environment is conditional on genetic risk.

Considerable interest has been generated by studies of first- and second-generation migrants. A meta-analysis of incidence studies found an even greater increase in psychosis risk for the second generation $(\mathrm{RR}=4.5,95 \% \mathrm{CI} 1.5-13.1)$ than for the first ( $R R=2.7,95 \%$ CI 2.3-3.2) (CantorGraae \& Selten, 2005). Further subgroup comparisons showed greater effect sizes for migrants from lower and middleincome versus high income countries and a remarkably high risk for migrants from countries where the majority of the population is Black $(\mathrm{RR}=4.8,95 \% \mathrm{CI}$ 3.7-6.2). It is important to note, however, that very high risks have also been noted for non-Black immigrant groups. Denmark has received many immigrants from its former colony Greenland and the risk for second-generation Greenlanders, who are ethnic Inuit, is 12.4 (95\% CI 4.7-33.1) times the risk for ethnic Danes (CantorGraae \& Pedersen, 2007). In the Netherlands, the risk for second-generation Moroccan males is 6.8 times $(95 \%$ CI 3.3-14.1) the risk for Dutch males (Veling et al, 2006). Selective migration of genetically vulnerable individuals has been ruled out as the sole explanation for the increased risk in migrants (Cantor-Graae \& Selten, 2005).

A third risk factor for schizophrenia is low intelligence. A follow-up study of Swedish conscripts, for instance, showed that the risk for schizophrenia was strongly and linearly related to low IQ (Zammit et $a l, 2004)$. Subjects with an average IQ, for example, had a significantly greater risk of developing schizophrenia than those with an IQ of more than $126(\mathrm{RR}=1.3,95 \%$ CI 1.04-1.54).

It has been estimated that the use of cannabis and other dopamine-enhancing drugs approximately doubles the risk (Arseneault et al, 2004). Hearing impairment and deafness are well-established risk factors for psychosis, but the magnitude of the relative risk for narrow schizophrenia associated with these factors is not precisely known (Thewissen et al, 2005). Finally, accumulating evidence indicates that a history of physical or sexual abuse during upbringing is a risk factor for schizophrenia, but opinions are still divided as to whether it is a causal risk factor (Read et al, 2005).

\section{SOCIAL DEFEAT}

Previously, we have proposed that the longterm experience of social defeat, defined as a subordinate position or as outsider status, may well be the common denominator for these findings (Selten \& Cantor-Graae, 2005). This interpretation is compatible with the high levels of competition in urban areas, the fewer career opportunities for people with low IQ, the social exclusion experienced by immigrants and people with 
hearing impairments and the humiliation of being abused.

The pattern of findings among immigrants supports this idea. A greater effect size for the second generation than for the first can be expected, because it is more humiliating to feel unwelcome in the country that you are born in than to feel unwelcome because you are born abroad. Furthermore, the immigrants with the highest risks (Moroccan males in the Netherlands, the Inuit in Denmark and African-Caribbeans in the UK) belong to the least successful and/or the most heavily discriminated groups. Moroccan males in the Netherlands are known for the highest crime and unemployment rates and have reported the highest frequency of the experience of discrimination. Remarkably, the schizophrenia risk for first- or second-generation Moroccan females is not increased (Veling et al, 2006). They have an inferior position in Moroccan society, but receive many opportunities for education and career in the Netherlands. Since migration has conferred upon them a considerable rise in social status, their low risk accords with the hypothesis. (Moroccans of either gender are exposed to the stress of acculturation and this type of stressor is therefore unlikely to explain the pattern of findings.) In Denmark, first- and second-generation Greenlanders have higher rates of schizophrenia than most other ethnic groups. It should be noted that Greenlanders and citizens from other Nordic countries are granted automatic entry into Denmark by inter-Nordic agreement, yet the rates for schizophrenia among Greenlanders are much higher than for persons from the other Nordic countries (Cantor-Graae et al, 2003). Rates of unemployment and social welfare benefits are higher among Greenlanders than among nearly all other immigrant groups, suggesting that social exclusion may play a key role in their increased risks for schizophrenia. Since the pain of social exclusion and humiliation may be mitigated by social support, the social defeat hypothesis also predicts a smaller risk increase in groups known for their strong social and family networks. Asian immigrants to the UK and Turkish immigrants to the Netherlands may serve as an example. The observation that the incidence in minority ethnic groups is smaller when they comprise a greater proportion of the local population accords with this view (Boydell et al, 2001).
The experience of social defeat will lead to a greater need for illicit drugs and to a greater susceptibility to these substances (see below). Finally, since males may feel more pressed to achieve a social rank than females, the hypothesis may also explain the higher risk and earlier onset in males (Aleman et al, 2003), but this is uncertain.

\section{CONCEPTUAL ISSUES}

The experience of defeat is in the eye of the beholder. Consequently, the absence of an association between socio-economic status of the parents and risk for schizophrenia in the child does not necessarily argue against the hypothesis. Children of high socio-economic status, who may feel pressured to meet the high expectations of their parents, may feel more easily defeated than other children. Furthermore, social defeat is neither a necessary nor a sufficient condition for the development of schizophrenia, is not always followed by the development of a psychiatric disorder and is also a risk factor for depression and addiction. Other factors, including those under a strong genetic control, would determine the nature of the outcome of exposure to social defeat. The important consideration here is that the genetic vulnerability to schizophrenia may be present in $10-20 \%$ of the population. Thus, the likelihood that gene carriers may go on to develop the schizophrenia phenotype may be strongly influenced by the experience of defeat. The importance of social defeat for the development of major psychiatric disorders is not surprising, given the central role of social competition in the evolutionary process.

Finally, it is worthwhile to note that 'social defeat' is also a consequence of a schizophrenic disorder, even before the emergence of psychotic symptoms. One may argue that it is difficult to distinguish between the episodes of defeat that constitute causal risk factors for the disorder, and the social decline that has already been set in motion and is part-element of the schizophrenia prodrome. The fact remains, however, that defeated populations (i.e. certain immigrant groups) produce more cases than non-defeated populations. The question as to whether defeat in particular or stress in general contributes to the aetiology of schizophrenia is difficult to answer at this point in time, because stressful experiences are also, to a varying degree, humiliating. The current hypothesis remains the most viable interpretation of the available epidemiological data.

\section{DOPAMINE FUNCTION IN SCHIZOPHRENIA}

Evidence for the role of dopamine in the development of schizophrenia is provided by the increased occupancy of striatal D2 receptors by dopamine in patients who have not received medication, the psychotogenic effects of dopamine-enhancing drugs and the known mode of action of antipsychotic drugs, i.e. blockade of dopamine D2 receptors (reviewed by Laruelle, 2003). Furthermore, current evidence indicates that the mesolimbic dopamine system is sensitised in schizophrenia. Sensitisation is a process whereby exposure to a given stimulus results in an enhanced response at subsequent exposures, in this example excess release of dopamine or the development of psychotic symptoms. The notion that patients with schizophrenia show dopamine sensitisation is supported by neuroreceptor imaging studies which have shown that amphetamineinduced dopamine release is increased in neuroleptic-naive individuals with schizophrenia; and many patients display increased sensitivity to the psychotogenic effects of illicit drugs. This means that they develop psychotic symptoms after exposure to doses that do not induce psychosis in healthy subjects. However, dopamine only mediates psychosis. Thus, important questions remain concerning the nature of the earlier events that lead to dopaminergic dysregulation. That social defeat could well be one of these earlier events is illustrated by a series of animal experiments.

\section{ANIMAL STUDIES}

In non-human primates dopamine function and social dominance are related. A neuroreceptor imaging study, which examined dopamine function in individually and socially housed cynomolgus macaques, yielded intriguing findings (Morgan et al, 2002). While the monkeys did not differ during individual housing, subsequent social housing increased the amount or availability of dopamine D2 receptors in the dominant monkeys and produced no change in the subordinate monkeys. Furthermore, the defeated monkeys consumed more cocaine than the dominant ones. The results indicated that individually housed monkeys and socially subordinate 
animals had relatively high levels of synaptic dopamine (i.e. dopaminergic hyperactivity) and that the dominant animals, after social housing, returned to a 'normal' state of dopamine function.

An even more interesting animal model for social defeat stress is the residentintruder paradigm, whereby a male rodent (the intruder) is put into the cage of another male (the resident). Within a minute the resident attacks the intruder and prompts him to display submissive behaviour. The experiment showed that social defeat stress leads to dopaminergic hyperactivity in the mesocorticolimbic system, not in the nigrostriatal system, and that the effects depended on the housing conditions after defeat (Tidey \& Miczek, 1996). Lengthy isolation after defeat amplified the changes in the dopaminergic activity, whereas return to the group mitigated them (Isovich et al, 2001). (The reader may note a parallel with the protective effects of social cohesion and high ethnic density.) The researchers also found that repeated experiences of defeat lead to a long-lasting behavioural sensitisation, in which the animal displays an enhanced behavioural and dopamine response to dopamine agonists (e.g. Covington \& Miczek, 2001). Several studies have confirmed this finding and shown that the endogenous kappa opioid system (McLaughlin et al, 2006) and the brain-derived neurotrophic factor (BDNF) (Berton et al, 2006) contribute to the development of the response.

These observations lead to the important conclusion that patients with schizophrenia who are antipsychotic-naive resemble in some aspects defeated animals and, subsequently, to the hypothesis that the experience of social defeat is one of the factors that can lead to behavioural sensitisation in humans. Two other schizophrenia risk factors that have been shown to produce behavioural sensitisation in rats are repeated exposures to dopamineenhancing drugs (Vanderschuren \& Kalivas, 2000) and perinatal anoxia (Brake et al, 1997). Furthermore, an experimental lesion in the ventral hippocampus, produced during the neonatal period, leads to dopamine sensitisation in the adult rat (Lipska et al, 1993). Thus, behavioural sensitisation seems a common pathogenetic mechanism for several schizophrenia risk factors.

It is important to note that social defeat is not the only social stressor that can influence dopamine function in rats. Isolation rearing leads to elevated basal dopamine levels and to an enhanced amphetamineevoked dopamine release (Hall et al, 1998). Maternal deprivation, in contrast, leads to a diminished behavioural response to amphetamine, despite apparent increases in presynaptic dopamine function in the nucleus accumbens (Hall et al, 1999).

Finally, since the gene for brain-derived neurotrophic factor is a candidate gene for schizophrenia, one can hypothesise that variation in this gene contributes to variation in the genetic vulnerability for schizophrenia by influencing the response to social defeat.

\section{TESTING THE HYPOTHESIS}

Thus, a major task for research in humans is to examine whether social defeat stress leads to sensitisation of the mesolimbic dopamine system. The results may have wide implications for our understanding of all major psychiatric disorders. Several strategies are possible; the most fruitful one for testing the hypothesis would be a prospective, longitudinal study examining dopamine function before and after a possible defeat. For example, one could compare healthy young adults who leave the same school and start competing in the labour market and re-examine them 2 years later. Since the experience of defeat is likely to lead to a decrease in self-esteem, one could conduct repeated measurements of self-esteem. This type of research has been facilitated by major advances in the development of implicit association tests to measure self-esteem (e.g., Greenwald \& Farnham, 2000; Greenwald et al, 2002). These tests assess automatic associations of self with positive or negative valence (by measuring differences in reaction times) and are less biased by the need to represent the self in a socially acceptable manner.

A second possibility is to compare, again, ethnic groups. It is likely that amphetamine-induced dopamine release is normally distributed and that the distribution in defeated populations is shifted towards the right. Consequently, one may compare immigrants from a 'super-high' risk group (e.g. second-generation Moroccan males in the Netherlands) to natives (Fig. 1).

Although this type of research currently has limited feasibility owing to the large numbers of participants that would be required to demonstrate a small- to medium-size difference in amphetamineinduced dopamine release between groups,

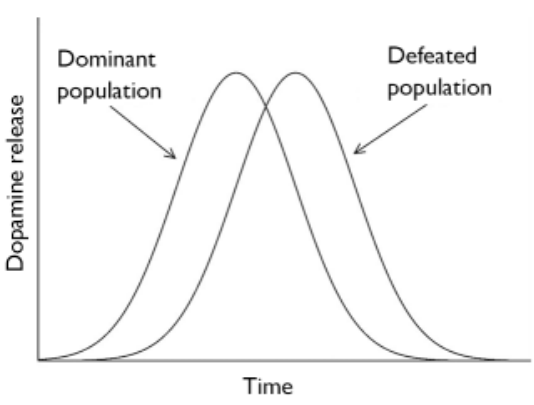

Fig. I Expected distribution of dopamine release, upon stimulation with amphetamine, in dominant and defeated population.

developments in technology may soon make this a more realistic undertaking.

Third, since the experience of social defeat is not only a risk factor for schizophrenia, but also for addiction and depression, one can examine the risks for these disorders in defeated populations. A study conducted in the Netherlands showed that those immigrant groups that are at increased risk of schizophrenia are also at an increased risk of drug use disorders, but not alcohol use disorders (Selten et al, 2007).

Finally, history carries out natural experiments. The social defeat hypothesis predicts the highest risks of schizophrenia for minority ethnic groups who are lowest on the social scale: Albanians in Greece, North-Africans in France, African-Americans in the USA, Ethiopian Jews in Israel. Importantly, if these groups are successful in other host countries, their risks should be not increased. Ethnic groups characterised by strong social and family networks (Cape Verdians in the Netherlands, for example) should have 'normal' risks or only mildly increased risks.

\section{REFERENCES}

Aleman, A., Kahn, R. S. \& Selten, J. P. (2003) Sex differences in the risk of schizophrenia: evidence from meta-analysis. Archives of General Psychiatry, 60, 565$57 \mathrm{I}$

Arseneault, L., Cannon, M., Witton, J., et al (2004) Causal association between psychosis and cannabis: examination of the evidence. British Journal of Psychiatry, 184, $110-117$.

Berton, O., McClung, C. A., DiLeone, R. J., et al (2006) Essential role of BDNF in the mesolimbic dopamine pathway in social defeat stress. Science, 3II, 864-868.

Boydell, J., van Os, J., McKenzie, K., et al (2001) Incidence of schizophrenia in ethnic minorities in London: ecological study into interactions with the environment. BMJ, 323, I-4. 
Brake,W. G., Boksa, P. \& Gratton, A. (1997) Effects of perinatal anoxia on the acute locomotor response to repeated amphetamine administration in adult rats. Psychopharmacology, 133, 389-395.

Cantor-Graae, E. \& Pedersen, P. (2007) Risk of schizophrenia in second-generation immigrants: a Danish population-based cohort study. Psychological Medicine, 37, 485-494.

Cantor-Graae, E. \& Selten, J. P. (2005) Schizophrenia and migration: a meta-analysis and review. American Journal of Psychiatry, 162, 12-24.

Cantor-Graae, E., Pedersen, C. B., McNeil,T. F., et al (2003) Migration as a risk factor for schizophrenia: a Danish population-based cohort study. British Journal of Psychiatry, 178, 367-372.

Covington, H. E. \& Miczek, K. A. (200I) Repeated social-defeat stress, cocaine or morphine. Effects on behavioral sensitization and intravenous cocaine selfadministration binges. Psychopharmacology, I58, 388-398.

Greenwald, A. G.. \& Farnham, S. D. (2000) Using the implicit association test to measure self-esteem and selfconcept. Journal of Personality and Social Psychology, 79, 1022-1038.

Greenwald, A. G., Banaji, M. R., Rudman, L. A., et a (2002) A unified theory of implicit attitudes,

stereotypes, self-esteem and self-concept. Psychological Review, 109, 3-25.

Hall, F. S., Wilkinson, L. S., Humby, T., et al (1998)

Isolation rearing in rats: pre- and post-synaptic changes in striatal dopaminergic systems. Pharmacology, Biochemistry and Behavior, 59, 859-872.

Hall, F. S., Wilkinson, L. S., Humby, T., et al (1999)

Maternal deprivation of neonatal rats produces enduring changes in dopamine function. Synapse, 32, 37-43.

Isovich, E., Engelmann, M., Landgraf, R., et al (200I) Social isolation after a single defeat reduces striatal dopamine transporter binding in rats. European Journal of Neuroscience, 13, 1254-1256.

Krabbendam, L. \& van Os, J. (2005) Schizophrenia and urbanicity: a major environmental influence

JEAN-PAUL SELTEN, MD, PhD, Department of Psychiatry, Rudolf Magnus Institute of Neuroscience, University Medical Centre Utrecht, Utrecht, the Netherlands, ELIZABETH CANTOR-GRAAE, PhD, Department of Health Science, Section for Social Medicine and Global Health, Lund University, University Hospital UMAS, Malmö, Sweden

Correspondence: Dr. J. P. Selten, Department of Psychiatry, University Hospital, P.O. Box 85500, 3508 GA Utrecht, The Netherlands. Email: j.p.selten@umcutrecht.nl

conditional on genetic risk. Schizophrenia Bulletin, 31, 795-799.

Laruelle, M. (2003) Dopamine transmission in the schizophrenic brain. In Schizophrenia (eds S. R. Hirsch \& D.Weinberger), pp.365-387. Blackwell.

Lipska, B., Jaskiw, G. E. \& Weinberger, D. R. (1993) Postpubertal emergence of hyperresponsiveness to stress and to amphetamine after neonatal excitotoxic hippocampal damage: a potential animal model of schizophrenia. Neuropsychopharmacology, 9, 67-75.

McLaughlin, J. P., Li, S., Valdez, J., et al (2006) Social defeat stress-induced behavioral responses are mediated by the endogenous kappa opioid system. Neuropsychopharmacology, 3I, I24I-1248.

Morgan, D., Grant, K. A., Gage, D., et al (2002) Socia dominance in monkeys: dopamine D2 receptors and cocaine self-administration. Nature Neuroscience, $\mathbf{5}$, 169-174.

Read, J., van Os, J., Morrison, A. P., et al (2005) Childhood trauma, psychosis and schizophrenia: a literature review with theoretical and clinical implications. Acta Psychiatrica Scandinavica, II2, 330-350.

Selten, J. P. \& Cantor-Graae, E. (2005) Social defeat: risk factor for schizophrenia? British Journal of Psychiatry, I87, $101-102$

Selten, J. P., Wierdsma, A., Mulder, C. L., et al (2007) Treatment seeking for alcohol and drug use disorders by immigrants to the Netherlands: retrospective, population-based cohort study. Social Psychiatry and Psychiatric Epidemiology, 42, 30I-306.

Thewissen, V., Myin-Germeys, I., Bentall, R., et a (2005) Hearing impairment and psychosis revisited. Schizophrenia Research, 76, 99-103.

Tidey, J.W. \& Miczek, K. A. (1996) Social defeat stress selectively alters mesocorticolimbic dopamine release: an in vivo microdialysis study. Brain Research, 72I, 140 149

van Oel, C. Sitskoorn, M. M., Cremer, M. P., et al (2002) School performance as a pre-morbid marker for schizophrenia: a twin study. Schizophrenia Bulletin, $\mathbf{2 8}$ $401-404$.

Vanderschuren, L. J. \& Kalivas, P. (2000) Alteration in dopaminergic and glutamatergic transmission in the induction and expression of behavioral sensitization: a critical review of preclinical studies. Psychopharmacology, I5I, 99-120.

Veling, W. A., Selten, J. P., Veen, N. D., et al (2006) Incidence of schizophrenia among ethnic minorities in the Netherlands: a four-year first-contact study. Schizophrenia Research, 86, 189-193.

Zammit, S., Allebeck, P., David, A. S., et al (2004) A longitudinal study of pre-morbid IQ score and risk of developing schizophrenia, bipolar disorder, severe depression, and other non-affective psychoses. Archives of General Psychiatry, 61, 354-360. 